\title{
О СПЕЦИФИКЕ ПРИМЕНЕНИЯ SMATH STUDIO В ЭЛЕКТРОТЕХНИЧЕСКИХ РАСЧЕТАХ
}

\section{SMATH STUDIO FEATURES APPLICATION IN ELECTRICAL ENGINEERING CALCULATIONS}

Yu. Ushakov

N. Ushakova

Summary. The article discusses the features of using the free mathematical program Smath Studio for solving electrical tasks. The differences between Smath Studio and Mathcad in the analysis of steady-state and transient modes in linear and nonlinear electrical and magnetic circuits, the specifics of graphic illustrations of electrical calculations, and the practical use of executable files are described.

Keywords: Smath Studio, Mathcad, theoretical electrical engineering, steady state and transient analysis.

\author{
Ушаков Юрий Александрович \\ К.т.н., доцент, Оренбургский государственный \\ университет \\ unpk@mail.ru \\ ушакова Наталья Юрьевна \\ К.т.н., доцент, Оренбургский государственный \\ университет \\ olaa56@mail.ru
}

Аннотация. В статье рассмотрены особенности применения бесплатной математической программы Smath Studio для решения электротехнических задач. Описаны отличия Smath Studio от Mathcad при анализе установившихся и переходных режимов в линейных и нелинейных электрических и магнитных цепях, специфика графических иллюстраций электротехнических расчетов, практическое использование исполняемых файлов.

Ключевые слова: Smath Studio, Mathcad, теоретическая электротехника, анализ установившихся и переходных режимов.

считать ее бесплатным клоном Mathcad было бы неверным. Это два разных приложения, имеющие свои характерные особенности, некоторые из которых являются эксклюзивными.

Smath Studio непрерывно дорабатывается, содержит постоянно пополняемый набор дополнений (плагинов), которые распространяются с открытым исходным кодом. С 2006 года по настоящее время было разработано около 60 стабильных версий программы, каждая последующая актуализированная версия содержит улучшения и исправления.

Одной из причин, по которой Smath Studio пока не столь популярен, как Mathcad, является недостаток разработанной подробной технической и методической документации по его применению. Значительная часть российских публикаций о Smath Studio носит либо обзорный характер [4; 5], либо содержит результаты решения какой-либо узкой задачи с использованием программы в качестве расчетного инструмента [6; 7]. Работ, содержащих комплексный анализ и рекомендации по использованию Smath Studio в конкретных профессиональных областях, довольно мало [8].

\section{Цель $\triangle$ анной работы}

Проанализировать функциональные возможности, специфику и целесообразность применения Smath 


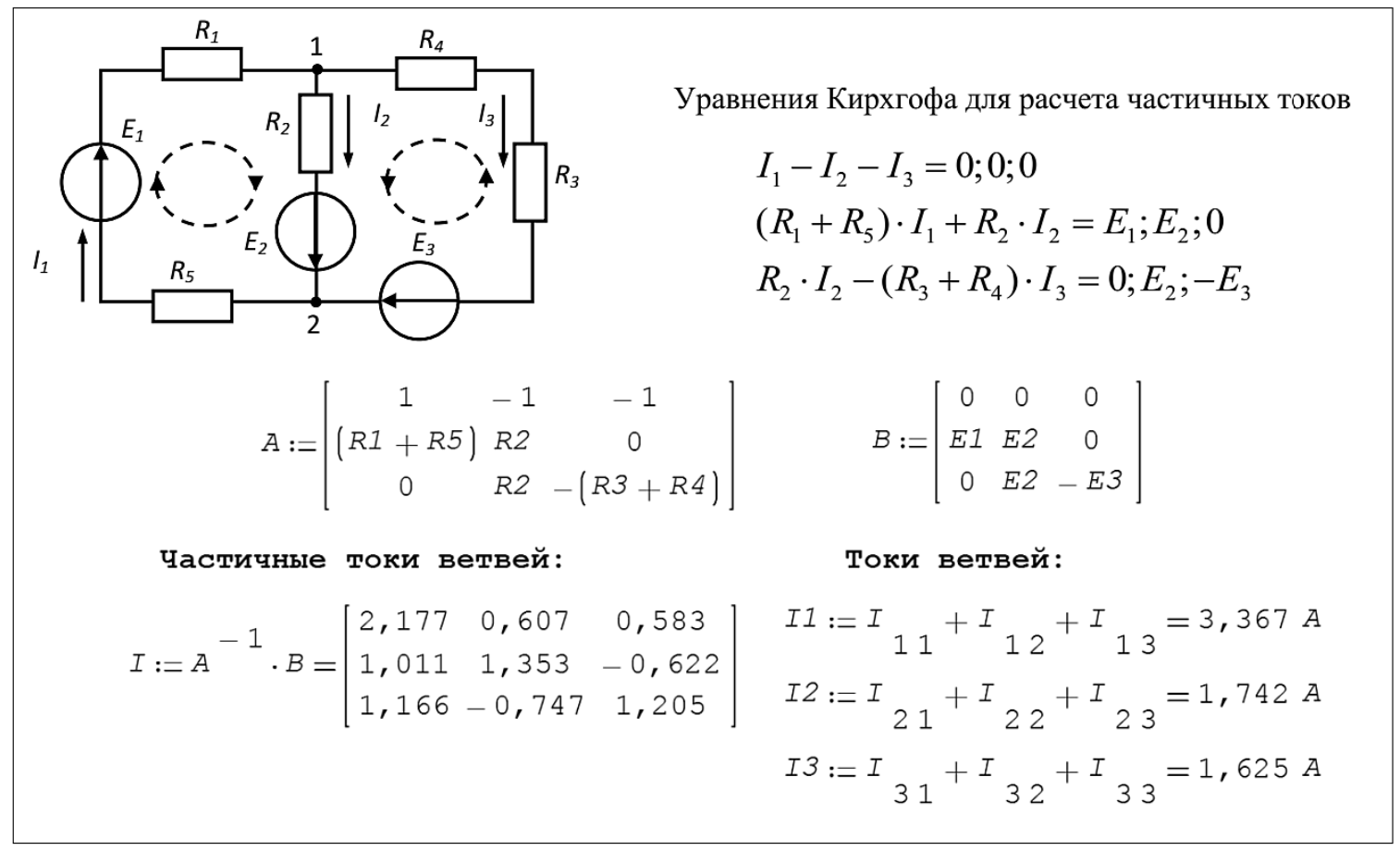

Рис. 1. Решение СЛАУ в методе наложения с тремя вариантами правой части уравнений

Studio как альтернативы Mathcad в электротехнических расчетах в области теоретической электротехники.

В математических моделях электротехнических устройств, используемых в теоретической электротехнике, применяется многообразный математический аппарат. Поэтому именно на примерах теоретической электротехники можно в комплексе оценить полноту и достаточность ресурсов Smath Studio, а также удобство его использования для решения широкого спектра электротехнических задач.

Значительная часть электротехнических расчетов базируется на теории электрических и магнитных цепей. Задачи теории цепей традиционно классифицируются по режимам работы цепи, по виду источников электромагнитной энергии, по характеру элементов цепи и их параметрам. Однако, чтобы всесторонне оценить преимущества и недостатки Smath Studio, более целесообразно применить к ним классификацию по применяемому при анализе цепей математическому аппарату, что и сделано в данной работе.

\section{1. О расчете ^инейных режимов электрических шепей в Smath Studio}

Математические модели электрических цепей описываются уравнениями, составленными различными методами на основе основных законов электротехники.
В зависимости от режима работы и параметров цепи эти уравнения могут быть линейные и нелинейные, алгебраические и дифференциальные, с действительными и комплексными коэффициентами.

Анализ установившихся режимов работы линейных электрических цепей постоянного и синусоидального тока сводится, как правило, к решению систем линейных алгебраических уравнений (СЛАУ) с действительными или комплексными коэффициентами.

Вычислительные возможности Mathcad, применяемые в электротехнике для решения таких систем, весьма разнообразны: метод обратной матрицы, функции lsolve, solve, вычислительный блок Given-Find [9]. При этом любым из перечисленных способов можно решать системы уравнений, как с действительными, так и с комплексными коэффициентами, что позволяет использовать их для анализа цепей как с постоянными, так и с гармоническими сигналами.

Функционал Smath Studio, используемый для решения СЛАУ, намного скромнее и, кроме того, зависит от характера корней уравнения.

Для цепей постоянного тока, описываемых уравнениями с действительными коэффициентами, решить СЛАУ в Smath Studio можно двумя путями:

- методом обратной матрицы; 
- с помощью функции roots, которая в программе есть в двух модификациях: roots (3) с указанием приближенных значений искомых неизвестных и roots (2) без указания приближений.

Для цепей синусоидального тока, уравнения которых имеют комплексные коэффициенты, возможности решения СЛАУ в Smath Studio ограничиваются вообще одним методом обратной матрицы, так как функция roots не предназначена для решения уравнений с комплексными корнями.

Функцией solve в Smath Studio в отличие от Mathcad можно решать только отдельные уравнения, а не систему, да и то они должны обязательно иметь действительные, а не комплексные корни.

Следует отметить, что индексация элементов вектора решения в Smath Studio при использовании метода обратной матрицы начинается с единицы, а не с нуля, как в Mathcad, что удобно при анализе электрических цепей.

Отличительной особенностью Smath Studio является его способность решать СЛАУ методом обратной матрицы сразу для нескольких вариантов правой части уравнений, в Mathcad такой возможности нет. В электротехнике эту способность Smath Studio можно использовать для расчета электрической цепи методом наложения. При расчете токов ветвей этим методом левые части уравнений, составленных для цепи, например, по законам Кирхгофа остаются неизменными, а вариантов правой части будет столько, сколько в цепи источников электромагнитной энергии. Каждый вариант правой части записывается при расчете в отдельный столбец в матрице свободных членов. Матрица результатов расчета будет содержать столько же столбцов. При расчете токов каждый столбец - это частичные токи ветвей, вызванные каждом источником энергии в отдельности.

На рисунке 1 приведен фрагмент расчета токов электрической цепи методом наложения с одновременным решением СЛАУ с тремя вариантами правой части уравнений.

\section{2. О реализашии символического метола расчета электрических чепей и использовании еАиниШ измерения B Smath Studio}

Анализ установившихся режимов линейных однофазных и трехфазных цепей синусоидального тока проводится традиционно символическим методом, при котором синусоидальные токи и напряжения заменяются их комплексными изображениями. Работа с комплексными числами в Smath Studio во многом аналогична работе с ними в Mathcad. Мнимая единица здесь тоже обозначается буквой $\boldsymbol{i}$, но возможности переобозначить ее в настройках программы через пункты Меню на принятое в электротехнике обозначение $\boldsymbol{j}$ (как это можно сделать в Mathcad) здесь не предусмотрено.

\section{Результаты}

расчетов токов и напряжений в цепях синусоидального тока получаются в виде комплексных чисел в алгебраической форме записи. Для перевода их в показательную форму записи нужно рассчитать модуль и аргумент комплексного числа. Как и в Mathcad, аргумент в Smath Studio по умолчанию считается в радианах, для перевода его в угловые градусы нужно вставить любым способом единицу измерения «Градус». Правда следует отметить, что в Smath Studio разработаны специальные функции $\boldsymbol{x y} 2 \boldsymbol{p o l}$ и pol2xy, которые должны облегчить пользователю перевод комплексного числа из алгебраической в показательную форму записи и обратно. Но работают они не всегда корректно. Например, у функции $\boldsymbol{x y 2 p o l}$ результат расчета аргумента выдается в радианах, а при попытке перевести его в градусы, на множитель 180/п автоматически умножаются рассчитанные и модуль, и аргумент, что приводит в итоге к неверным результатам.

Кроме того, в Smath Studio нет опции получения из комплексного числа комплексно-сопряженного числа. В электротехнике так называемый «сопряженный комплекс тока» широко используется для расчета полной комплексной мощности. Для его нахождения можно предложить пользовательскую функцию

$$
\dot{I}_{\text {sopr }}=\frac{|\dot{I}|^{2}}{\dot{I}} .
$$

Smath Studio позволяет при вводе исходных данных вводить и их единицы измерения, обширный список которых предлагается во вкладке Меню «Вставка». При расчетах программа должна их распознать и автоматически подставить нужные единицы измерения в результаты расчетов. Во многих простых задачах электротехники эта опция действительно работает правильно и ее можно использовать. Но в некоторых случаях наличие введенных единиц измерения мешает и не позволяет провести расчеты. Например, при расчете цепи по законам Кирхгофа с помощью функции roots или методом обратной матрицы программа выдает сообщение об ошибке «Единицы измерения не соответствуют» и не считает токи. Это связано с разной размерностью уравнений Кирхгофа и соответственно строк матрицы. В подобных ситуациях лучше проводить расчеты в безразмерных величинах. 


\section{Исходные данные:}

$$
\begin{aligned}
& E:=300 \mathrm{~B} \quad R:=10 \text { OM } \quad L 1:=30 \cdot 10^{-3} \Gamma_{\mathrm{H}} \\
& L 3:=20 \cdot 10^{-3} \text { ГH } \quad C 2:=60 \cdot 10^{-6} \Phi \quad T:=0,4 \cdot 10^{-2} \mathrm{C}
\end{aligned}
$$

Расчет токов ветвей методом двух узлов:

$$
\begin{aligned}
& j:=\sqrt{-1} \quad f:=\frac{1}{T}=250 \text { Гц } \quad \omega:=\left\lfloor 2 \cdot \pi \cdot f \mid=1570 \frac{\text { pад }}{\mathrm{c}}\right. \\
& \varphi 1:=\frac{E \cdot \frac{1}{R+j \cdot \omega \cdot L 1}}{\frac{1}{R+j \cdot \omega \cdot L 1}+\frac{1}{R-j \cdot \frac{1}{\omega \cdot C 2}}+\frac{1}{R+j \cdot \omega \cdot L 3}}=(20,24-100,903 \cdot \text { i }) \mathrm{B} \\
& I 1:=\frac{-\varphi 1+E}{R+j \cdot \omega \cdot L 1}=(3,257-5,248 \cdot i) \mathrm{A} \\
& |I 1|=6,177 \AA \quad \arg (I 1)=-58,18^{\circ}
\end{aligned}
$$

Некорректная работа функции ху2pol:

$$
\text { xy2pol }(3,191 ;-5,379)=\left\{\begin{array}{l}
6,254 \\
-1,035
\end{array} \quad \operatorname{xy2pol}(3,191 ;-5,379)=\left\{\begin{array}{l}
358,344 \\
-59,322
\end{array} .\right.\right.
$$

田

\section{Расчет мощностей :}

Сопряженный комплекс тока (пользовательская функция)

I1 sopr $:=\frac{|I 1|^{2}}{I 1}=(3,257+5,248 \cdot i) \mathrm{A}$

Пользовательские единицы измерения

$$
\mathrm{BA}:=\mathrm{BT} \quad \mathrm{BAp}:=\mathrm{BT}
$$$$
S:=E \cdot I 1 \text { sopr }=(976,982+1574,482 \cdot \text { i }) \mathrm{B} \mathrm{A}
$$$$
P:=\operatorname{Re}(S)=976,982 \text { Вт }
$$$$
Q:=\operatorname{Im}(S)=1574,482 \mathrm{BAp}
$$

Векторная диаграмма токов

$$
I:=\left[\begin{array}{llllll}
0 & \text { I1 } & 0 & \text { I2 } & 0 & \text { I3 }
\end{array}\right]^{\mathrm{T}}
$$$$
\text { токи:= augment }(\operatorname{Re}(I) ; \operatorname{Im}(I))
$$

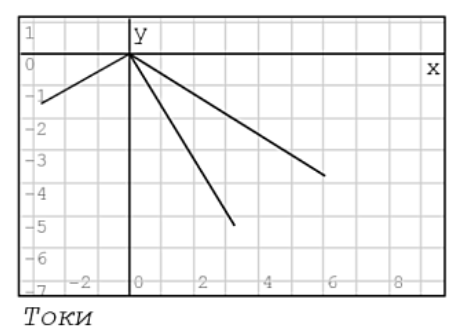

Рис. 2. Использование в расчете электрической цепи пользовательских функций и единиц измерения

Следует отметить, что в приведенном в Меню Smath Studio списке единиц измерения отсутствуют единицы для ряда применяемых в электротехнических расчетах величин: угловой частоты, реактивной и полной мощностей. Для таких величин целесообразно вводить в рас- четах при необходимости пользовательские единицы измерения.

На рисунке 2 показан фрагмент расчета разветвленной цепи синусоидального тока с использовани- 


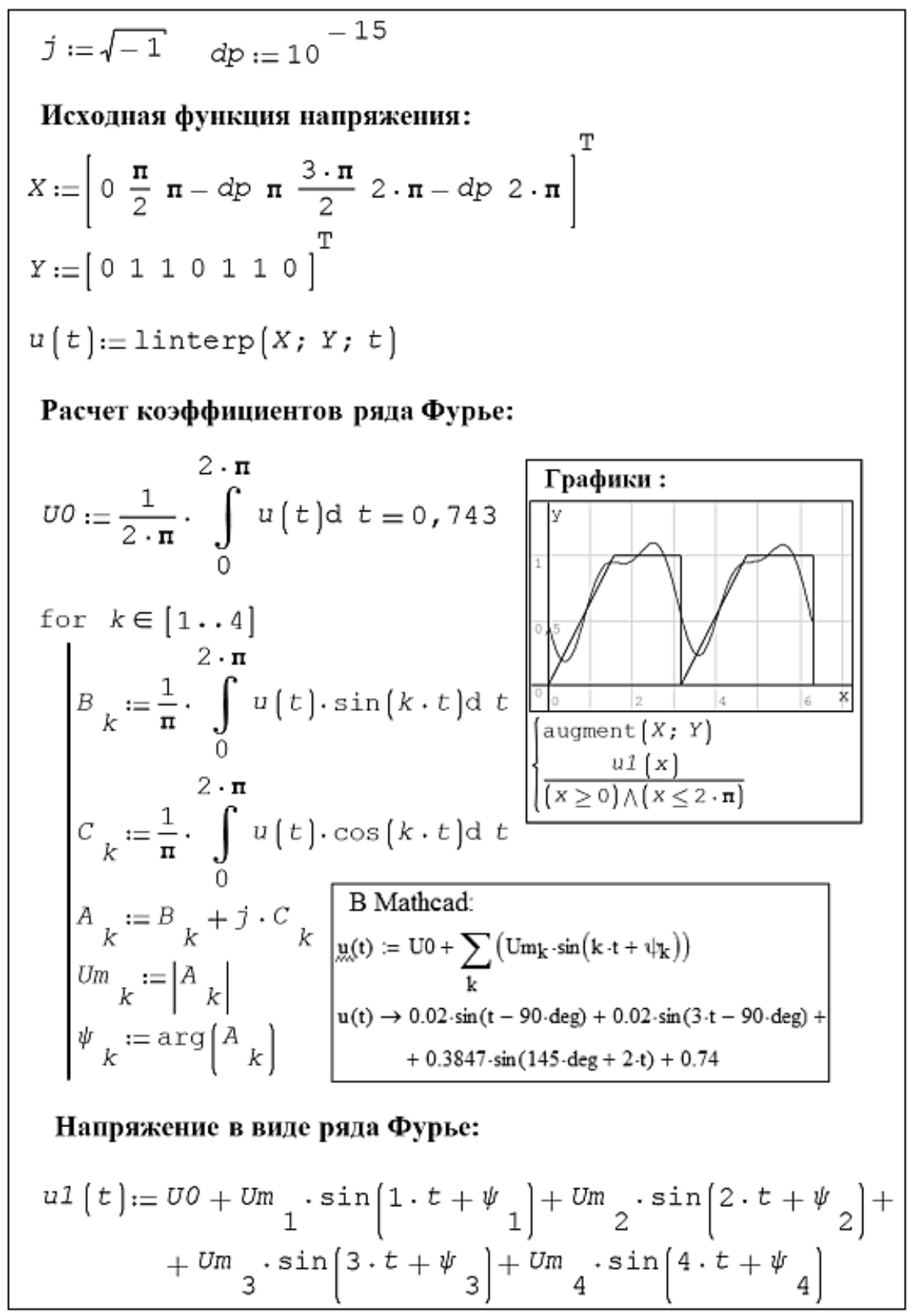

Рис. 3. Разложение несинусоидального напряжения в ряд Фурье

ем встроенных и пользовательских единиц измерения и функций.

При расчете результаты практически во всех пунктах автоматически получились с верными единицами измерения, независимо от сложности формулы. Пользовательские единицы измерения вставлены у угловой частоты $\omega$, а также у полной $(\boldsymbol{S})$ и реактивной $(\boldsymbol{Q})$ мощностей (изначально программа поставила у угловой частоты единицу измерения Герц, а у обеих мощностей - Ватт).

Для вставки единиц измерения полной и реактивной мощностей ( $B A$ и $B A p$ ) была переопределена имеюща- яся в программе единица мощности $B m$. После этого пользовательские единицы $B A$ и $B A p$ появились в списке единиц измерения Меню, что позволило использовать их в дальнейших расчетах, но только в данном документе.

Пользовательскую составную единицу измерения $p a d / c$ таким способом вставить нельзя, программа выдает ошибку «Выражение задано неверно». Поэтому единица измерения угловой частоты была просто скомпилирована из имеющихся в списке Меню отдельных единиц измерения (радиан, секунда). В списке единиц измерения Меню она при таком способе вставки 
не появляется и носит чисто иллюстративный характер.

В приведенном на рисунке 2 фрагменте расчета показана также пользовательская функция для расчета сопряженного комплекса тока, используемого далее в формуле полной комплексной мощности $\boldsymbol{S}$. Кроме того продемонстрированы некорректные результаты работы функции $\boldsymbol{x y 2 p o l ~ н а ~ п р и м е р е ~ р а с ч е т а ~ м о д у л я ~ и ~ а р г у м е н т а ~}$ комплексного тока $\boldsymbol{I}_{l}$. Сначала аргумент тока рассчитан в радианах, при попытке перевести его в градусы изменился не только аргумент, но и модуль тока.

Во фрагменте расчета часть формул скрыта с помощью элемента «Область» из вкладки Меню «Вставка».

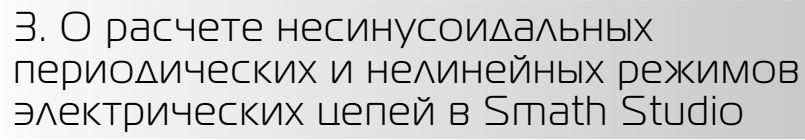

При анализе установившихся режимов в электрических цепях несинусоидального тока используется более сложный математический аппарат. Расчет таких цепей основан на принципе наложения и включает в себя: разложение периодической несинусоидальной функции в ряд Фурье; расчет цепи для каждой гармоники ряда; запись результирующих величин токов и напряжений в виде суммы мгновенных значений (в виде ряда Фурье).

B Smath Studio для расчета коэффициентов ряда Фурье функцию можно задать только двумя способами:

- аналитическим выражением;

- векторами с последующей интерполяцией.

B Mathcad, кроме этого, для задания функции можно еще использовать логическую функцию if. B Smath Studio при таком задании функции она не воспринимается в дальнейших операциях расчета коэффициентов ряда Фурье.

Первый способ целесообразно использовать, если несинусоидальную функцию достаточно просто описать аналитическими выражениями на определенных отрезках. Задание функции векторами наиболее удобно использовать для формализации графиков, заданных отрезками прямых. При необходимости моделирования скачка такой функции можно изменить значение одной или нескольких координат на такую малую величину, что это не отразится на дальнейших вычислениях.

Расчет коэффициентов ряда Фурье проводится по общеизвестным в высшей математике формулам. Для этих целей в Smath Studio есть функция вычисления определенного интеграла. Следует отметить, что в SMath Studio в отличие от Mathcad можно вычислять только определенные интегралы. Расчет можно проводить отдельно для каждой гармоники, а можно оформить в виде циклического процесса, используя оператор for и элемент line с панели Программирование.

На рисунке 3 приведен пример разложения в ряд Фурье функции, заданной отрезками прямых. Для интерполяции функции использована функция линейной интерполяции linterp.

В этом примере показаны некоторые «странности» Smath Studio:

- для того чтобы рассчитать модули и аргументы гармоник напряжения, пришлось присвоить им наименования $(\boldsymbol{U} \boldsymbol{m} \boldsymbol{k}, \boldsymbol{\Psi} \boldsymbol{k})$, так как в противном случае программа выдавала сообщения об ошибках: «Аргумент должен быть скаляром» и «Ошибка приведения типов». Mathcad такие расчеты провел бы без переопределения величин;

- нельзя записать формулу напряжения в мгновенной форме записи, используя знак суммы (как это показано во фрагменте расчета из Mathcad на рисунке 3). Выражение напряжения в виде суммы гармоник записано вручную, но и в этом случае Smath не смог подставить в него числовые данные, как это делает Mathcad, чтобы получилось читаемое выражение.

Еще одна «странность» Smath Studio обнаружилась при попытке разложить в ряд Фурье функцию, заданную аналитическими выражениями на определенных отрезках периода. Программа выдает ошибку при расчете коэффициентов ряда, если в формуле общий множитель $1 / \pi$ умножается на сумму определенных интегралов. Если же на этот множитель умножается каждый интеграл, расчет реализуется успешно.

При анализе нелинейных электрических и магнитных цепей, в которых нелинейные характеристики элементов В основном задаются совокупностью табличных значений широко используется интерполяция. Smath Studio позволяет использовать помимо линейной интерполяции интерполяцию сплайнами. При этом следует учитывать, что на заданном отрезке при любой интерполяции получаются близкие значения функции, а вот за пределами заданных отрезков данных значения при линейной интерполяции и интерполяции сплайнами будут сильно различаться.

На рисунке 4 показан фрагмент расчета магнитной цепи, в котором уравнение Кирхгофа для интерполированных функций магнитных потоков решается с помощью функции solve и находится значение межузлового магнитного напряжения. 


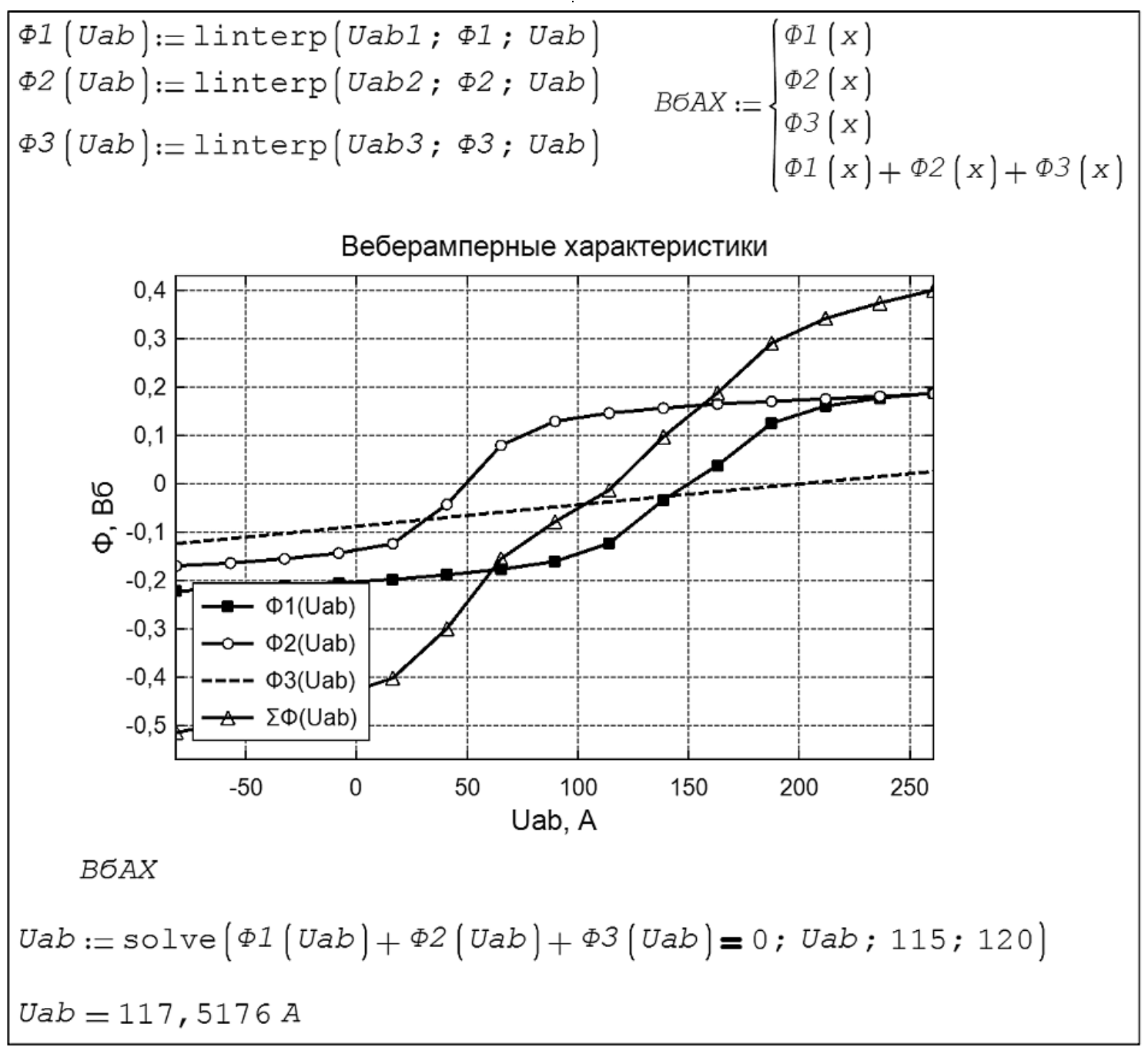

Рис. 4. Фрагмент графоаналитического расчета магнитной цепи

\section{Oсобенности Smath Studio при анализе перехонных прочессов в электрических чепях}

Электрическое состояние линейных электрических цепей в переходных режимах описывается обыкновенными дифференциальными уравнениями. В электротехнике для их решения наиболее часто применяются классический и операторный методы. Оба этих метода имеют четкие алгоритмы решения, для реализации которых в Smath Studio имеется необходимый, но не всегда достаточный функционал.

Одним из этапов классического метода расчета переходного процесса является составление характеристического уравнения и нахождение его корней.Дляцепей, содержащихболее одного накопителя энергии (конденсатора или катушки индуктивности), уравнение имеет вторую или более степень.
В зависимости от параметров пассивных элементов электрической цепи характеристическое уравнение может иметь как действительные, так и комплексносопряженные корни. Именно при наличии комплексных корней в Smath Studio возникают неожиданные проблемы в расчетах переходных процессов.

Первая из них связана с ограниченной символьной математикой программы. Решить характеристическое уравнение с комплексно-сопряженными корнями в Smath Studio можно, используя только функцию polyroots. Для формирования вектора коэффициентов характеристическое уравнение, которое для разветвленной электрической цепи, как правило, записывается в виде многочлена, должно быть предварительнопреобразовано изаписано какполином. B Mathcad любые преобразования сложных выражений выполняются легко с использованием разнообразного набора функций символьной математики. B Smath Stu- 
dio, к сожалению, преобразовать сложный многочлен к требуемому виду, используя имеющиеся символьные операции, практически невозможно. Проще выполнить преобразования вручную или в какой-либо другой программе.

Вторая проблема при комплексно-сопряженных корнях в классическом методе расчета переходного процесса может возникнуть на этапе расчета постоянных интегрирования. В случае комплексных корней постоянные интегрирования находятся из системы нелинейных алгебраических уравнений, для решения которых в Smath Studio используется функция roots. В данном случае целесообразно использовать ее модификацию roots (3), в записи которой указываются приближения искомых величин. Однако если заданные приближения отличаются даже незначительно от истинных значений, решение не будет найдено. Mathcad при решении подобных систем уравнений не столь критичен к задаваемым начальным приближениям.

Операторный метод расчета переходных процессов заключается в том, что при расчётах действительные функции времени $\boldsymbol{f}(\boldsymbol{t})$, описывающие процессы в электрических цепях и называемые оригиналами, заменяют их операторными изображениями $\boldsymbol{F ( p )}$ с помощью преобразования (интеграла) Лапласа. Электрическая схема заменяется операторной схемой замещения, из которой рассчитываются изображения искомых токов и напряжений, далее от изображений переходят к оригиналам.

Следует отметить, что возможности Smath Studio позволяют так же, как и в Mathcad, рассчитать операторные токи и напряжения в виде функций или получить решение в общем виде, используя оператор символьного вычисления.

Для перехода от изображений к оригиналам в символьных операциях Mathcad имеется оператор invlaplace. Правда, справедливости ради нужно отметить, что корректно этот оператор работал до версии Mathcad 13 включительно. В версиях Mathcad 14 и 15 для обратного преобразования Лапласа сложные выражения приходится раскладывать на более простые, используя операции символьной математики [10].

B Smath Studio оператора процедуры обратного преобразования Лапласа нет, поэтому для перехода от изображений к оригиналам целесообразно использовать стандартный алгоритм используемой в электротехнике теоремы разложения. И здесь успешность расчета опять зависит от того, какие корни получатся в процессе расчета: действительные или комплексно-сопряженные. При комплексных корнях возникают описанные выше неудобства при формировании полинома.

Кроме того, и при действительных, и при комплексных корнях в Smath Studio не получается сформировать читаемые результирующие выражения для оригиналов и их приходится формировать вручную, используя промежуточные результаты расчетов.

Переходной процесс в электрической цепи можно рассчитывать и численно. Для численного решения дифференциальных уравнений в Smath Studio можно применять дополнение «ODE Solvers» из онлайнгалереи, которое содержит функции rkfixed и Rkadapt. Обе функции используют для решения метод Рунге Кутты четвертого порядка, rkfixed - с фиксированным шагом, Rkadapt - с адаптивным шагом. На рисунке 5 показан фрагмент расчета переходного процесса в сложной электрической цепи с двумя накопителями энергии с помощью функции rkfixed.

\section{5. О графических особенностях Smath Studio в электротехнических расчетах}

Расчет электрических цепей в электротехнике практически всегда сопровождается построением двумерных графиков временных, векторных или топографических диаграмм. Нужно отметить, что подход к заданию и построению графиков в Smath Studio радикально отличается от Mathcad.

Одно из своеобразных требований Smath Studio заключается в том, что при построении двумерного графика функции одного переменного на поле графика эта переменная должна быть обязательно обозначена латинской буквой «Х», при любом другом символе программа напишет, что он «не определен». В самом же выражении функции, которая записана до графика, эта переменная может быть обозначена любой другой буквой, на построение графика это никак не повлияет. Причем это требование распространяется только на функции, заданные аналитически.

Если же функция задана в виде двух векторов значений по осям абсцисс и ординат, то для построения ее графика такой оба вектора должны быть объединены в единую матрицу. И в этом случае безразлично, какими буквенными символами обозначены вектор аргументов и вектор функции. Название объединенной матрицы тоже может быть произвольным, и даже не обязательно должно быть записано в виде функции. В электротехнике именно так задаются вектора токов и напряжений для их изображения на комплексной плоскости. Примеры и особенности построения графиков функций, заданных аналитически и векторами приведены выше на рисунках 2 и 3. 


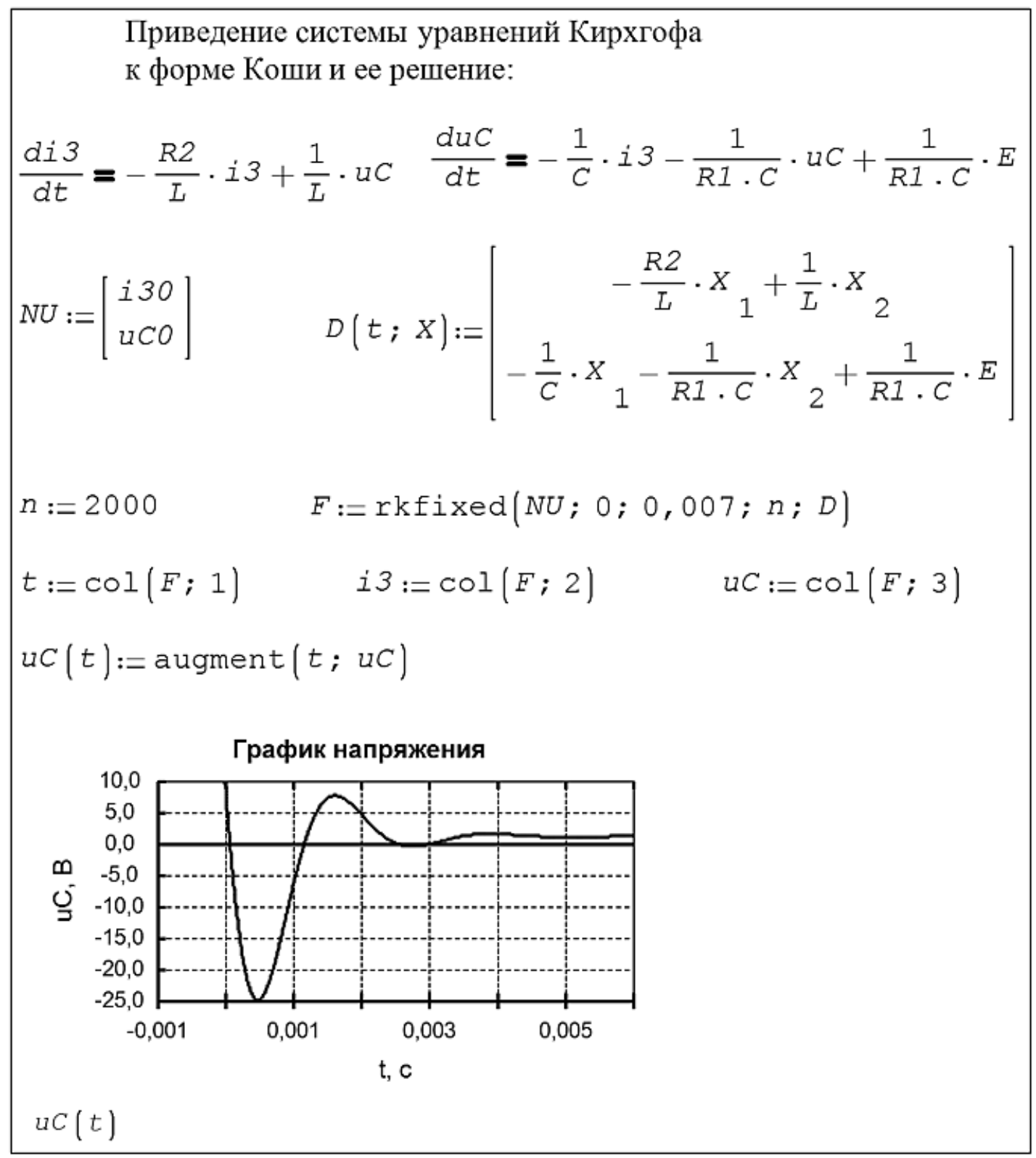

Рис. 5. Численный расчет переходного процесса

К основному недостатку базовой версии Smath Studio при построении графиков в сравнении с Mathcad относится невозможность задать пределы изменения аргумента перед построением графика. Независимо от пределов, в которых строится график, пределы осей на полотне графика вставятся всегда одинаковые (от -12 до +12 по оси «Х» и от -8 до +8 по оси «у»). Если график меньше или больше этих пределов, полотно можно масштабировать только вручную колесиком мыши и клавишами Shift и Ctrl. Mathcad же пределы осей устанавливает автоматически в зависимости от строящейся функции. Кроме того, в Smath Studio вообще не предусмотрена функция полноценного форматирования самих графиков: нельзя поменять их цвет и толщину, вставить пользовательские названия осей, легенду и т.п.

Для построения графика аналитической функции на заданном участке можно использовать в записи функции элементы программирования или булевы функции. Такая возможность показана на одном из графиков рисунка 3. К сожалению, эти действия только ограничивают участок, на котором изобразится функция, но пределы осей не изменяют, и график после построения, если он выходит за стандартные пределы полотна, все равно приходится масштабировать. 


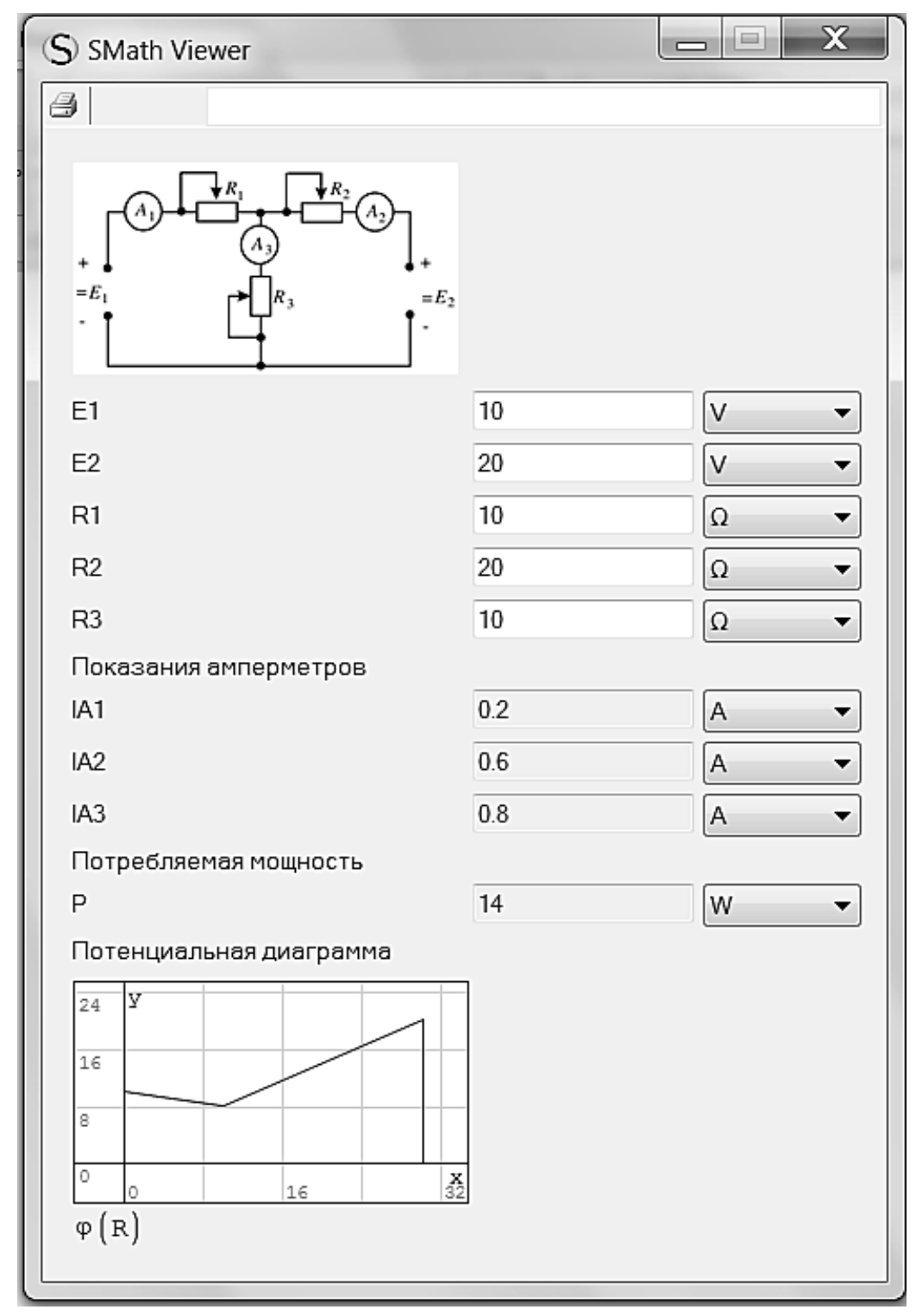

Рис. 6. Исполняемый файл в формате ехе

Чтобы расширить возможности оформления графиков целесообразно установить из онлайн галереи Smath Studio соответствующие дополнения (плагины). В списке галереи есть несколько плагинов с близким функционалом: «X-Y Plot Region», «ZedGraph Region», «NPlot Region». Из них дополнение «X-Y Plot Region» имеет наиболее привычное и краткое меню с набором большинства необходимых для оформления инженерных графиков функций: можно устанавливать пользовательские пределы осей, изменять сетку, линии графика, шрифты, вставлять дополнительные оси, названия графика, осей, легенду и т.д. Некоторые возможности графических дополнений показаны на рисунках 4 и 5. Графики веберамперных характеристик на рисунке 4 построены в дополнении
«X-Y Plot Region», график переходного напряжения на рисунке 5 построен с использованием плагина «ZedGraph Region». Ни одно из упомянутых графических дополнений не русифицировано, что создает некоторые неудобства при работе с ними.

\section{6. О совместимости с Mathcad и исполняемых файлах}

Возможность открытия в Smath Studio или сохранения документа в формате файла Mathcad на первый взгляд кажется очень заманчивой. Ведь это позволяет пользователюиспользоватьмногочисленныепредыдущие наработки в Mathcad по расчету электротехнических устройств. Однако следует учитывать, что при открытии 
в Smath Studio документа в формате «xmcd» откроются только выражения, в которых есть функции, аналогичные функциям Smath Studio. Оригинальные процедуры программа проигнорирует или вообще покажет ошибку открытия документа. Аналогичная ситуация будет и при открытии в Mathcad документа из Smath Studio, сохраненного в формате «xmcd».

Особо следует остановиться на документах, сохраняемых в Smath Studio в формате автономных исполняемых файлов ехе. Это оригинальная способность программы. На официальном сайте Smath Studio раздел, в которомприводится описаниетаких файлов называется «Smath Viewer» (Smath Телезритель). Приложение Viewer можно очень эффективно использовать для виртуальных лабораторных работ по электротехнике или в какихлибо исследовательских целях.

Пример лабораторной работы в приложении Viewer показан на рисунке 6.

Пользователь может изменять параметры схемы, при этом автоматически изменятся токи ветвей, мощность, график потенциалов. Для того чтобы пользователь видел только задаваемые параметры и результаты расчета или график, в расчетном файле фрагмент с расчетом «спрятан» в Области из вкладки Меню «Вставка».
В расчетных файлах, планируемых к сохранению в формате exe, можно вставлять рисунки и графики, единицы измерения, использовать элементы управления (флажок, кнопку, движок, список) из соответствующих плагинов. В итоге можно получить очень интересные исполняемые файлы, позволяющие исследовать и анализировать работу электротехнических устройств при изменении их параметров, конфигурации, режима работы.

\section{Зак^ючение}

Ограниченный объем статьи и приведенного графического материала не позволил продемонстрировать все возможности и особенности бесплатной математической программы Smath Studio. Помимо рассмотренных выше программа имеет характерные отличия ввода и корректировки данных и выражений, интересный функционал для оформления расчетной работы непосредственно в программе, облачную и мобильную версию.

Однако даже рассмотренный спектр задач позволяет сделать вывод, что с учетом некоторых указанных в статье недостатков Smath Studio можно эффективно использовать в решении большинства инженерных и научных прикладных задач в сфере электротехники.

\section{ЛИТЕРАТУРА}

1. SMath Studio: официальный сайт программы._ URL: https://ru.smath.com (дата обращения 08.11.2020)

2. Приказ Минкомсвязи России «0б утверждении методических рекомендаций по переходу государственных компаний на преимущественное использование отечественного программного обеспечения, в том числе отечественного офисного программного обеспечения» // сайт Минцифры России, 2019._URL: https://digital.gov.ru/ru/documents/6294 (дата обращения 08.11.2020)

3. Аверкин C. SMath Studio. Краткое руководство [Электронный ресурc].—URL: https://ru.smath.com/o6зор/c1be00e3-eb8c-78a5-b1f9-f6e15457ecbc/peзюмe (дата обращения 08.11.2020)

4. Потапов Л. А. Выполнение научно-технических расчетов с помощью свободно распространяемых программ/ Л. А. Потапов, М.Л. Потапов // Вестник Брянского государственного технического университета.— 2016. — № 1(49).—C. 140-145.

5. Бобровских А. В. Свободное программное обеспечение в высшихучебных заведениях военной направленности. Математические продукты [Электронный ресурс] / А. В. Бобровских, Т. Ю. Урывская, А. П. Алимов // Инженерный вестник Дона._ 2019.— № 9._ URL: ivdon.ru/ru/magazine/archive/N9y2019/6246 (дата обращения 08.11.2020 г.)

6. Кольцов И.В.Аспекты применения программных продуктов для моделирования технологических процессов/ И.В. Кольцов // Информационные технологии в УИС. - 2019.—№ 3.- С. 49-57.

7. Нуждин Л.В. Методы численного моделирования системы «свайный фундамент - грунтовое основание» в условиях сейсмических воздействий/ Л. В. Нуждин, В. С. Михайлов, В. В. Резяпкин // Природные и техногенные риски. Безопасность сооружений.— 2019.—№ 5 (42). — C. $28-34$.

8. Корницкая М.Н. Обучение студентов строительных специальностей младших курсов информационным технологиям на примере отечественной математической системы Smath Studio| М. Н. Корницкая, А. И. Распутина, И. А. Макаревич// Ползуновский альманах.— 2018. № 1.—C. 109-115.

9. Исаев Ю. Н. Практика использования системы MathСad в расчетах электрических и магнитных цепей. / Ю. Н. Исаев, А. М. Купцов.—- Москва: СОЛОН-ПРЕСС, 2017. - $180 \mathrm{c}$.

10. Ушакова Н. Ю. Анализ линейных электрических цепей в стационарных и переходных режимах: учебное пособие/ Н. Ю. Ушакова, Л. В. Быковская.Москва: «ТиРу», 2012. - 133 с. 